\title{
Norois
}

Environnement, aménagement, société

$226 \mid 2013$

Commerce et développement local, déplacements urbains, valorisations agricoles, découpages électoraux

\section{Les équipements du quotidien en France : un facteur d'attractivité résidentielle et de développement pour les territoires?}

Equipment daily in France: a factor of residential attractiveness and local development?

\section{Magali Talandier et Valérie Jousseaume}

\section{(2) OpenEdition} Journals

Édition électronique

URL : https://journals.openedition.org/norois/4525

DOI : 10.4000 /norois. 4525

ISBN : 978-2-7535-2287-9

ISSN : $1760-8546$

\section{Éditeur}

Presses universitaires de Rennes

\section{Édition imprimée}

Date de publication : 30 mars 2013

Pagination : $7-23$

ISBN : 978-2-7535-2285-5

ISSN : 0029-182X

Référence électronique

Magali Talandier et Valérie Jousseaume, « Les équipements du quotidien en France : un facteur d'attractivité résidentielle et de développement pour les territoires ? », Norois [En ligne], 226 | 2013, mis en ligne le 30 mars 2015, consulté le 13 janvier 2022. URL : http://journals.openedition.org/norois/ 4525 ; DOI : https://doi.org/10.4000/norois.4525 


\title{
Les équipements du quotidien en France : un facteur d'attractivité résidentielle et de développement pour les territoires?
}

\author{
Equipment Daily in France: A Factor of Residential Attractiveness and Local Development?
}

\author{
Magali Talandier ${ }^{* a}$, Valérie Jousseaume ${ }^{b}$
}

\footnotetext{
* Auteur correspondant

a PACTE - UMR 5194 CNRS/IEP/UPMF/UJF (Université Joseph Fourier de Grenoble), Institut de Géographie

Alpine, 14 bis av. Marie Reynoard - 38100 Grenoble (magali.talandier@ujf-grenoble.fr)

b ESO-Nantes, UMR 6590 ESO (Université de Nantes), Institut de Géographie et d'Aménagement (IGARUN),

Campus du Tertre, BP 81227 - 44312 Nantes cedex 3 (valerie.jousseaume@univ-nantes.fr)
}

Résumé : À l'heure où le renouveau rural et l'expansion des petites villes questionnent les enjeux de coûts sociaux et environnementaux liés aux mobilités régulières des individus... au moment où les urbanistes, chercheurs, décideurs se concentrent sur la re-densification des grandes métropoles... cet article affirme d'abord que les mobilités quotidiennes des Français sont complexes et finalement assez mal connues. Cet article propose de considérer l'offre d'équipements banals comme un révélateur de ces mobilités quotidiennes. Les résultats suggèrent que les petites villes et les gros bourgs-centres (compris entre 3000 et 20000 habitants) sont proportionnellement aussi bien armés que les villes moyennes et grandes en matière de centralités des mobilités de consommation quotidienne. Cet article montre également que la présence de ces équipements est un facteur de la capacité des territoires à attirer de nouvelles populations et à faire circuler la richesse. Les équipements, commerces et services ordinaires, nœuds de nos mobilités quotidiennes, participeraient donc au développement territorial durable, conjuguant économie productive, économie résidentielle et économie présentielle, duquel les campagnes ne sont pas exclues.

\begin{abstract}
Many worry about the rural renew or demographic expansion of small towns (social and environmental impacts of commuting). In a context of willingness density city by city planners, researchers, this paper proposes to observe and analyze the centrality of daily mobility of population. This paper shows the complexity of the French daily mobility. Analysis of these mobilities is made from ordinary supply of equipment. This work shows that small towns and large town centers (between 3,000 and 20,000 inhabitants) are proportionally as well equipped as medium and large cities. Finally, this study explains that the presence of this equipment is a factor for the territories to attract new residents. Facilities, shops and services would participate in the sustainable territorial development, combining residential economy and productive economy, which the campaigns are not excluded.
\end{abstract}

Mots clés : petites villes - espace rural - développement local - économie résidentielle - économie présentielle - consommation - services de proximité - mobilité

Keywords: small towns - rural areas - consumption - local services - résidential économy - mobility 


\section{INTRODUCTION}

Les rythmes s'accélèrent, les distances parcourues augmentent, les mobilités se complexifient. La désynchronisation des temps produit une ville malléable (Gwiazdzinski, 2007) et une certaine plasticité des territoires. Dans ce contexte, tenter de mesurer ce qui bouge, surtout quand ça bouge de plus en plus vite et de façon de plus en plus aléatoire n'est pas aisé. Il paraît vain de chercher à repérer les régularités « du quotidien ». Pour autant, on ne peut pas parler de dislocation des systèmes territoriaux. Les points d'ancrage - certes multiples - demeurent.

Les travaux actuels sur ces sujets sur-valorisent les grandes villes. Les études et les réponses préconisées reposent trop souvent sur des représentations discutables de ce que sont nos mobilités quotidiennes, elles-mêmes étant conditionnées par les données statistiques existantes en matière de déplacements. Ainsi, la plupart des zonages statistiques sont construits à partir des seules navettes quotidiennes d'actifs occupés entre leur domicile et leur travail. C'est le cas des aires urbaines, mais aussi des bassins de vie de l'INSEE. Dans le cas des bassins de vie, le critère d'accès à ce que l'INSEE nomme les biens intermédiaires ${ }^{1}$ est également pris en compte (Blanc et al., 2007). Ces définitions et délimitations statistiques - fort utiles par ailleurs influencent incontestablement nos représentations des « territoires vécus ${ }^{2}$ » par les populations, mais également les discours et politiques en matière de centralité urbaine, de forme urbaine, de périurbanisation. Pourtant, $60 \%$ de la population ${ }^{3}$ n'appartient pas à la catégorie population active occupée et n'a donc pas comme contrainte de mobilité quotidienne celle des déplacements domicile-travail. Parmi les actifs occupés, au moins $20 \%$ sont des travailleurs mobiles $^{4}$ (Davezies et al., 2008). Ainsi, en 2008, l'en-

1. Notons que la notion de biens intermédiaires recouvre des équipements comme l'hôpital, les lycées, les opéras, les piscines couvertes. En raison des conventions de calculs, la localisation de ces équipements influe bien plus sur la délimitation des bassins de vie que la localisation des médecins généralistes, de l'école, de la boulangerie, du café, de la pharmacie.

2. La carte de l'INSEE représentant les territoires vécus était construite à partir du zonage en aire urbaine et emploi de l'espace rural et de l'inventaire communal concernant la consommation de biens et services intermédiaires.

3. Ou bien encore $30 \%$ des ménages.

4. Les travailleurs mobiles : actifs occupés exerçant une profession qui peut les contraindre à faire des «tournées » de clientèle et non un seul trajet domicile-travail par jour (infirmières, services aux personnes, artisans dans le bâtiment, etc.). semble des déplacements liés au travail (notion plus large que le seul trajet domicile-travail) représentent $24 \%$ des kilomètres parcourus annuellement par les Français, tous types de déplacements confondus ${ }^{5}$ (CGDD, 2010). Plus généralement, comme l'exprime J. Viard (2004), nous passons moins de $10 \%$ de notre vie à travailler. Le temps libre est devenu notre premier temps de vie.

On peut prendre également l'exemple des enquêtes ménages-déplacements (EMD) réalisées et disponibles dans la plupart des grandes agglomérations françaises. Ces enquêtes ne concernent que les déplacements à moins de 100 km réalisés du lundi au vendredi, hors vacances. Ces enquêtes ignorent donc $40 \%$ des déplacements annuels (en $\mathrm{km}$ ) qui se font à plus de $100 \mathrm{~km}$ (Orfeuil, 2008), mais également une partie non négligeable de nos déplacements, réalisés le week-end.

À l'heure où la pensée dominante se concentre sur la grande ville "post-carbone » (compacte, durable), il semble intéressant de questionner les centralités quotidiennes de la population française, en dehors de la seule mobilité domicile-travail, d'étudier la place que jouent les différents échelons urbains ou les simples communes rurales et d'en analyser l'impact sur le développement des territoires. Entre mobilités multiples et ancrage du quotidien, notre objectif est de tenter de repérer et d'analyser la « centralité des quotidiens ». Il ne s'agit plus d'une centralité individuelle régulière, mais d'une centralité collective qui, à notre sens, structure encore - voire de plus en plus - les territoires. Ce serait la persistance de la proximité, dans une société devenue « hypertexte » pour reprendre l'expression de F. Ascher (2009). Nous faisons donc l'hypothèse que, dans ce monde de flux et de mouvements, la centralité des quotidiens demeure un point d'ancrage à des échelons très fins géographiquement, des populations résidentes et présentes ${ }^{6}$. Cette présence d'équipements serait un facteur d'attractivité auprès de nouvelles populations, permettrait d'accélérer la circulation de la richesse. Ce serait donc un des leviers majeurs du développement durable

\footnotetext{
5. Si l'on ne considère que les déplacements locaux (déplacements à moins de $80 \mathrm{~km}$ un jour de semaine ouvré pour des individus de six ans ou plus), les trajets domicile-travail représentent un peu moins de $20 \%$ des mobilités quotidiennes. L'ensemble des trajets liés au travail représente $40 \%$ des kilomètres parcourus lors de ces déplacements locaux.

6. Population présente sur un territoire sans nécessairement y être résidente et donc recensée, comme les touristes (C. Terrier et al., 2005), mais également les excursionnistes, ou encore les actifs au lieu de travail...
} 
de notre territoire national, développement dont ne serait nullement exclu l'espace rural.

\section{IDENTIFIER LES NGUDS DE NOS MOBILITÉS QUOTIDIENNES}

L'absence de données en matière de suivi des déplacements quotidiens dans les petites villes, les bourgs-centres et les campagnes, où il n'existe pas d'enquêtes déplacements des ménages spécifiques, rend l'exercice difficile, mais pas impossible. Ne disposant pas d'enquêtes de mobilité assez fines pour mener une analyse comparative sur ces organismes urbains de petite taille, nous avons privilégié une approche méthodologique par l'offre. Étudier ces mécanismes de mobilité à travers l'offre de biens et services et non à travers la demande nous semble, en réalité, tout aussi pertinent, voire même plus enrichissant.

En effet, cette offre révèle finalement très bien la demande collective globalement non intermittente, bien qu'individuellement discontinue, aléatoire, fluctuante ou saisonnière. Il ne s'agit donc pas de tenter d'observer, de mesurer les mobilités quotidiennes individuelles dans les villes, ou encore dans l'espace rural, mais d'étudier la matérialisation de cette centralité des quotidiens malgré leurs temporalités diffuses et éclatées.

\section{Les équipements comme indicateurs de la centralité des quotidiens}

L'objet de cette partie est de repérer ce qui crée à des niveaux très quotidiens, de la centralité commune, collective, partagée. L'approche n'est pas donc pas celle de la rareté des biens et services offerts. Il ne s'agit pas non plus de tenter de définir une nouvelle hiérarchie urbaine, ni de contribuer au débat sur cette notion ${ }^{7}$. L'objet de ce travail n'est pas non plus de définir une géographie du commerce, de s'interroger sur sa place dans et hors la ville comme cela a pu être développé récemment (Gasnier, 2010 ; Dugot et Pouzenc, 2010) ou bien encore sur l'impact des commerces dans les espaces fragiles (Soumagne, 2002). Nous cherchons simplement à

7. Le lecteur intéressé par ces notions pourra consulter l'abondante littérature des années 1980 sur ce sujet comme par exemple (Allen et Sanglier, 1979; Kayser, 1972; Laborie, 1979) ou bien plus récemment (Pumain, 1992, 1994, 2004), ou bien encore des travaux de l'INRA (Hilal et al., 1995). observer la présence d'équipements ordinaires, quotidiens - ces nœuds banals de nos mobilités - et surtout d'en analyser l'impact sur les dynamiques territoriales.

Notre méthode se fonde sur la base permanente des équipements (BPE) de l'INSEE, sur l'année 2009. Cette base est destinée à inventorier annuellement, le niveau d'équipements et de services rendus par commune, à la population. Le champ actuel recouvre les domaines des services, marchands ou non, des commerces, de la santé et de l'action sociale, de l'enseignement, des sports et loisirs et du tourisme. Elle recense près de deux millions d'équipements répartis sur le territoire national (échelle communale), ventilés en plus de 150 catégories. L'INSEE propose trois gammes de « services » différentes. Après regroupement d'équipements proposant les mêmes services ${ }^{8}$, puis suppression des équipements touristiques ${ }^{9}$ destinés, selon l'INSEE, à une population non résidente, l'INSEE rassemble les équipements sur la base de leur co-présence dans les communes françaises, repérée grâce à la méthode des «nuées dynamiques ${ }^{10}{ }^{»}$. L'organisme d'études statistiques propose de distinguer la gamme de proximité, la gamme intermédiaire et la gamme supérieure (annexe 1).

Cette typologie en trois gammes aurait pu être reprise telle quelle dans ce travail. Néanmoins, il nous a semblé préférable de partir des données brutes mises à disposition sur le site de l'INSEE, afin de réintroduire certains équipements touristiques dans notre analyse ${ }^{11}$ et surtout pour tenter de mieux qualifier cette notion de «centralité des quotidiens » qui désigne les points de convergence et d'ancrage des mobilités des populations dans ce travail. Les regroupements d'équipements communaux ou intercommunaux des écoles maternelles, élémentaires ont été conservés, ainsi que les regroupements concernant les lycées (lycées d'enseignement général distingués des lycées professionnels).

8. Par exemple regroupement des écoles maternelles et des classes maternelles de regroupement pédagogique intercommunal dispersé, ou encore d'épicerie et supérette..

9. Domaine skiable, centre équestre, baignade aménagée, port de plaisance, boucle de randonnée, agence de voyage, hôtel et camping homologués, information touristique.

10. Système de classification des données quantitatives.

11. Parce que ces équipements participent aussi en partie aux mobilités des populations dans leurs pratiques récréatives régulières ou non. Nous avons cependant, comme l'INSEE, exclu les boucles de randonnée de notre analyse qui nous paraissent d'une autre nature que les équipements de la base. 
Nous privilégions une approche territoriale afin de repérer tout simplement les équipements géographiquement les plus fréquents dans les communes de la France métropolitaine, équipements dont on peut supposer qu'ils sont les expressions matérielles des sociabilités et des mobilités les plus quotidiennes. Ces équipements d'usage quotidien sont définis ici par leur plus ou moins grande présence sur le territoire national. Ils se différencient des autres services par leur niveau de couverture territoriale. Il est intéressant de noter que la gamme de proximité proposée par l'INSEE correspond à un seuil minimum de $15 \%$ de communes équipées, alors même que la méthodologie de construction des gammes repose sur une toute autre méthode que la nôtre. Nous retiendrons ce seuil de $15 \%$, qui correspond en effet à une certaine césure dans les équipements proposés (annexe II). Au sein de ces équipements d'usage quotidien, deux types sont à distinguer :

- ceux qui se caractérisent par la mobilité du fournisseur du service (11 équipements voire dans ce cas 11 services, tels que le plombier ou l'infirmier) que nous qualifierons de services mobiles de proximité. Ces services ne sont pas associés à un point d'ancrage de nos flux de consommation quotidienne. Ici, c'est le fournisseur du service qui se déplace vers son client. Sa localisation dépend souvent de son lieu de domicile situé à proximité de sa zone de chalandise.

- ceux qui induisent un déplacement de l'usager ou du client et peuvent être qualifiés d'équipements fixes (21 équipements, comme l'école élémentaire ou la banque) que nous qualifierons d'équipements de centralité des quotidiens. L'ensemble de nos mobilités complexes, variées, aléatoires, font émerger des nœuds, des points de centralité collective qui seraient repérables grâce à ces données.

Cette dernière catégorie qui retiendra notre attention par la suite, dans la mesure où se concentrant dans les villes et bourgs, ils peuvent contribuer à l'ancrage des populations et constituer un facteur d'attractivité auprès de nouvelles populations. En tête des équipements de centralité des quotidiens, près de deux communes sur trois sont équipées d'une école élémentaire (tableau 1). Il faut noter également, la forte présence des restaurants (près d'une commune sur deux). Ce résultat souligne, certes l'attrait touristique de certaines communes, mais il souligne également la complexité de nos mobilités quotidiennes. Parmi les équipements très fréquents se trouvent les terrains de grands jeux (foot, rugby...), les boulodromes ou encore les terrains de tennis... qui constituent eux aussi des lieux banals de sociabilité. On trouve ensuite le coiffeur, le garagiste, la boulangerie, la Poste, la pharmacie, etc., soit autant de traces de convergence de nos mobilités collectives, individuellement aléatoires et complexes.

Les services mobiles de proximité les plus usuels comprennent tous les métiers de la construction (maçon, plombier, menuisier, peintre/plâtrier, électricien...), les infirmiers, les agents immobiliers, les kinésithérapeutes (comme les médecins, ces trois dernières services peuvent être acteurs de centralité ou de proximité), les taxis, etc. Au-dessous du seuil de $15 \%$, on trouve les hôtels ${ }^{12}$, les écoles de conduite, les maisons de retraite, les orthophonistes, les teinturiers, les regroupements pédagogiques intercommunaux, les magasins de meubles, les magasins d'articles de sport, soit des équipements de centralité plus occasionnelle, ou de gamme intermédiaire pour reprendre la terminologie officielle (annexe 2).

Enfin, la gamme appelée « supérieure » par l'INSEE correspond à une présence des équipements dans moins de $5 \%$ des communes françaises. En dessous du seuil de $5 \%$, on observe une catégorie de services plus rares, plus coûteux à construire également, exigeant des économies d'échelle. Ce sont, par exemple, les piscines couvertes, les gendarmeries, les médecins spécialistes, les hôpitaux, les lycées, etc., jusqu'aux centres de lutte contre le cancer qui sont identifiés comme les équipements les plus rares de la BPE 2009 (24 en France).

Les équipements de centralité des quotidiens regroupent 22 types d'équipements différents et totalisent sur l'espace national près de 800000 équipements, soit 43,6\% des équipements recensés dans la BPE.

Neuf services mobiles de proximité sont identifiés, qui représentent $28,5 \%$ des équipements de la base nationale de 2009.

Les équipements intermédiaires sont au nombre de 41 et pèsent pour 20,9\% des équipements. Enfin, les équipements plus rares - appelés supé-

12. Les hôtels non pris en compte par l'INSEE sont présents dans 15,7\% des communes métropolitaines, nous les incluons dans les équipements intermédiaires. 
Tableau 1 : Les équipements présents dans plus de $15 \%$ des communes françaises (Source : INSEE, BPE $2009-$ calculs M. Talandier)

Shops and services in more than $15 \%$ of French municipalities

\begin{tabular}{|c|c|c|c|}
\hline Nom & $\begin{array}{c}\mathrm{Nb} \text { de } \\
\text { communes } \\
\text { équipées }\end{array}$ & $\begin{array}{l}\text { Part des } \\
\text { communes } \\
\text { équipées } \\
(\%)\end{array}$ & Typologie des auteurs \\
\hline Ecole élémentaire & 22749 & 62,1 & Centralité des quotidiens \\
\hline Terrains de grands jeux & 18096 & 49,4 & Centralité des quotidiens \\
\hline Restaurant & 17452 & 47,7 & Centralité des quotidiens \\
\hline Réparation automobile et de matériel agricole & 15038 & 41,1 & Centralité des quotidiens \\
\hline Boulodrome & 14027 & 38,3 & Centralité des quotidiens \\
\hline Coiffure & 13605 & 37,2 & Centralité des quotidiens \\
\hline Tennis & 13580 & 37,1 & Centralité des quotidiens \\
\hline Boulangerie & 13480 & 36,8 & Centralité des quotidiens \\
\hline Salle non spécialisé & 11079 & 30,3 & Centralité des quotidiens \\
\hline Plateau extérieur ou salle multisports & 10356 & 28,3 & Centralité des quotidiens \\
\hline Bureau de poste & 9478 & 25,9 & Centralité des quotidiens \\
\hline Agence immobilière & 8889 & 24,3 & Centralité des quotidiens \\
\hline Pharmacie & 8368 & 22,9 & Centralité des quotidiens \\
\hline Epicerie & 8360 & 22,8 & Centralité des quotidiens \\
\hline \begin{tabular}{|l} 
Boucherie charcuterie \\
\end{tabular} & 8205 & 22,4 & Centralité des quotidiens \\
\hline Masseur kinésithérapeute & 8047 & 22,0 & Centralité des quotidiens \\
\hline Ecole maternelle & 7844 & 21,4 & Centralité des quotidiens \\
\hline Chirurgien dentiste & 6498 & 17,7 & Centralité des quotidiens \\
\hline Banque, Caisse d'Epargne & 6373 & 17,4 & Centralité des quotidiens \\
\hline Fleuriste & 6286 & 17,2 & Centralité des quotidiens \\
\hline Salle de terrains de petits jeux & 6223 & 17,0 & Centralité des quotidiens \\
\hline Soins de beauté & 6144 & 16,8 & Centralité des quotidiens \\
\hline Maçon & 19163 & 52,3 & Services mobiles de proximité \\
\hline Menuisier, charpentier, serrurier & 18559 & 50,7 & Services mobiles de proximité \\
\hline Plombier, couvreur, chauffagiste & 18048 & 49,3 & Services mobiles de proximité \\
\hline Plâtrier peintre & 15867 & 43,3 & Services mobiles de proximité \\
\hline Electricien & 15301 & 41,8 & Services mobiles de proximité \\
\hline Infirmier & 10291 & 28,1 & Services mobiles de proximité \\
\hline Médecin omnipraticien & 10118 & 27,6 & Services mobiles de proximité \\
\hline Taxi & 8356 & 22,8 & Services mobiles de proximité \\
\hline Entreprise générale du bâtiment & 7254 & 19,8 & Services mobiles de proximité \\
\hline
\end{tabular}

rieurs - regroupent 80 types d'équipements différents, mais ne représentent que $7 \%$ des équipements présents sur le territoire (tableau 2).

\section{Densité et diversité des équipements de la centralité des quotidiens}

À partir de ce classement des équipements, nous pouvons distinguer quels sont les niveaux d'équipement des communes et espaces géographiques français. Deux indicateurs sont proposés (tableau 3) :

- la densité, qui mesure le total des équipements pour 1000 habitants;

- la diversité, qui mesure le nombre d'équipements différents par catégorie de services.

Ainsi, lorsque dans une commune, on recense trois boulangeries, deux épiceries et un restaurant, on comptabilisera six équipements indicateurs de centralité des quotidiens. Ce nombre est ensuite divisé par le nombre d'habitants pour obtenir une densité pour 1000 habitants. Pour cette même commune, la diversité de centralité est de trois équipements. Le tableau 3 présente la densité et la diversité des équipements par type d'espaces INSEE selon le dernier zonage en aire urbaine de $2010^{13}$ et selon la taille démographique des communes : villages et bourgs $<3000$ hab. ; bourg-centre ou petite ville : de 3000 à 20000 hab.; ville moyenne : de 20000 hab. à 100000 hab.; grande ville : plus de 100000 habitants.

13. L'INSEE propose depuis 2012 un nouveau zonage de l'espace en aires urbaines, comprenant : Les grands pôles urbains : unité urbaine offrant 10000 emplois ou plus; Le périurbain composé des couronnes et communes multipolarisées par les grands pôles urbains (au moins 40 \% des actifs travaillent dans un ou plusieurs pôles ou dans des communes attirées par celui-ci); Les pôles moyens et leur couronne : unités urbaines offrant entre 5000 et 10000 emplois et leur périphérie (40\% des actifs travaillent dans le pôle moyen ou dans une commune attirée par celui-ci); Les petits pôles et leur couronne : unités urbaines offrant entre 1500 et 5000 emplois et leur périphérie (40\% des actifs travaillent dans le petit pôle ou dans une commune attirée par celui-ci); Les autres communes multipolarisées à savoir un rural sous influence des moyens et petits pôles; Les autres communes isolées de l'influence urbaine ou rural isolé. 


\begin{tabular}{|l|c|c|c|}
\hline Type d'équipements & $\begin{array}{c}\text { Nb d'équipements } \\
\text { différents }\end{array}$ & $\begin{array}{c}\text { Nb total } \\
\text { d'équipements }\end{array}$ & $\begin{array}{c}\% \text { des } \\
\text { équipements }\end{array}$ \\
\hline Centralité des quotidiens & 22 & 791350 & 43,6 \\
proximité & 9 & 517388 & 28,5 \\
Intermédaires & 41 & 380207 & 20,9 \\
Supérieurs & 80 & 127267 & 7,0 \\
\hline Total & 152 & $\mathbf{1 8 1 6 2 1 2}$ & $\mathbf{1 0 0 , 0}$ \\
\hline
\end{tabular}

Tableau 2 : Nombre d'équipements par catégorie de services (Source : INSEE, BPE 2009 - calculs M. Talandier)

of Service Number of Facilities by Type

\begin{tabular}{|c|c|c|c|c|c|c|c|c|c|}
\hline & \multicolumn{5}{|c|}{ Densité d'équipements pour 1000 hab. } & \multicolumn{4}{|c|}{ Diversité des équipements } \\
\hline & Total & $\begin{array}{c}\text { Centralité } \\
\text { des } \\
\text { quotidiens }\end{array}$ & $\begin{array}{l}\text { Services } \\
\text { mobiles de } \\
\text { proximité }\end{array}$ & Intermédiaires & & $\begin{array}{c}\begin{array}{c}\text { Centralité } \\
\text { des } \\
\text { quotidiens }\end{array} \\
\end{array}$ & $\begin{array}{l}\text { Services } \\
\text { mobiles de } \\
\text { proximité }\end{array}$ & Intermédiaires & Supérieurs \\
\hline \multicolumn{10}{|c|}{ 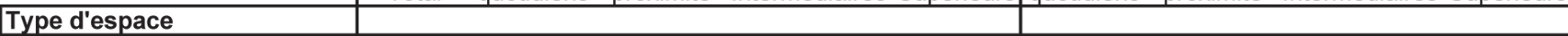 } \\
\hline Grand pôle urbain & 29,9 & 12,5 & 7,9 & 6,7 & 2,7 & 15,9 & 7,3 & $\overline{15,6}$ & 9,5 \\
\hline Périurbain & 24,3 & 11,4 & 8,4 & 3,8 & 0,7 & 6,3 & 3,3 & 2,5 & 0,5 \\
\hline Pôle moyen & 36,5 & 15,4 & 8,8 & 9,4 & 3,0 & 7,1 & 3,7 & 5,6 & 3,2 \\
\hline Petit pôle & 39,3 & 16,8 & 9,4 & 10,7 & 2,4 & 9,1 & 4,3 & 9,8 & 3,2 \\
\hline Rural ss influence urb. & 25,2 & 12,4 & 8,9 & 3,3 & 0,5 & 4,4 & 2,4 & 1,3 & 0,2 \\
\hline Rural isolé & 38,9 & 18,3 & 11,2 & 8,3 & 1,1 & 4,5 & 2,3 & 2,4 & 0,4 \\
\hline \multicolumn{10}{|l|}{ Taille des communes } \\
\hline Gandes villes & 34,8 & 14,9 & 8,2 & 7,9 & 3,7 & 21,9 & 9,0 & 39,8 & 64,6 \\
\hline Villes moyennes & 28,6 & 11,6 & 7,5 & 6,5 & 2,9 & 21,4 & 9,0 & 35,9 & 38,3 \\
\hline Petites villes (ou bourgs centre) & 30,1 & 12,7 & 8,2 & 7,2 & 2,0 & 19,7 & 8,4 & 22,0 & 8,4 \\
\hline Villages et bourgs & 26,5 & 12,6 & 9,1 & 4,0 & 0,7 & 5,2 & 2,8 & 1,8 & 0,3 \\
\hline Total & 29,3 & 12,8 & 8,3 & 6,1 & 2,1 & 6,6 & 3,4 & 3,8 & 1,4 \\
\hline
\end{tabular}

Tableau 3 : Densité et diversité des catégories d'équipements par type d'espace et de ville (Source : INSEE, Recensement 2009 - BPE 2009 - calculs M. Talandier)

Densité des équipements pour 1000 habitants = (nombre total d'équipements par catégorie/population 2007) x 1000 . En moyenne, en France, on compte 12,8 équipements de centralité des quotidiens pour 1000 habitants en 2009. Diversité des équipements = nombre d'équipements différents au sein d'une même catégorie. Les communes françaises sont, en moyenne, équipées de 6,6 services de centralité des quotidiennes sur 22 recensés. Density and diversity of services by type of cities and territories

Il est intéressant de souligner, qu'en termes de densité d'équipement pour 1000 habitants, il y a peu de différences entre l'urbain et le rural, ou encore entre les grandes, moyennes ou petites villes. La densité des équipements nœuds des centralités quotidiennes varie entre 11,4 et 18,3 équipements pour 1000 habitants. Cette variabilité somme toute assez faible révèle un sous-équipement relatif pour tous les équipements tant quotidiens, que services de proximité, qu'intermédiaires ou supérieurs, des espaces périurbains, puis du « périurbain rural » ou rural sous faible influence urbaine. On retrouve ici un résultat mis en évidence dans d'autres travaux (Talandier, 2005 ; Friedland et al., 2009), à savoir un déficit chronique et croissant des espaces périurbains en nombre d'équipements puisque la croissance démographique y est bien plus rapide que celle des équipements.

Les services mobiles de proximité, qui correspondent plus à la localisation du domicile des entrepre- neurs (artisans dans le secteur de la construction, infirmiers etc.), sont un peu moins présents, mais répartis de façon plus homogène entre les territoires. Sur le plan de la diversité des équipements proposés, les données sont différentes. La diversité de l'offre souligne le rôle de centres des grands pôles urbains tout d'abord, puis des petits pôles ruraux dans ces systèmes de flux. Ces résultats confirment les analyses des bourgs ruraux détaillées développées par V. Jousseaume dans l'Ouest français, ou encore des équipes de l'INRA (Jousseaume, 1996; INRA, 2008). Le périurbain présente une diversité de l'offre de services supérieure à celle des espaces ruraux. Le rural isolé, qui apparaissait à la lecture du premier indicateur comme un espace relativement bien pourvu, arrive en queue de classement. Relativement au nombre d'habitants, ces espaces peu denses semblent mieux équipés que la moyenne nationale, mais cet indicateur masque une réalité qualitative, celle de «la palette de choix » 
des services. Cette remarque vaut également pour les espaces ruraux sous faible influence urbaine, qui connaissent pourtant une croissance rapide de leur population. Ces décalages entre croissance démographique et diversité des équipements sont évidemment facteurs de mobilités quotidiennes. Il y a là d'ailleurs un certain paradoxe entre la volonté de réduire les mobilités et une tendance au regroupement des services, publics en particulier, dans les pôles urbains.

Appliqué à la taille des villes, l'indicateur de la diversité des équipements met en valeur le rôle éminemment structurant des villes. La différence est très importante entre les petites villes (communes de 3000 à 20000 habitants) et le reste des villages, tant en matière de consommation quotidienne, de circulation des richesses et de convergence des flux quotidiens. Les petites villes offrent une diversité de choix en matière de centralité des quotidiens et de services mobiles de proximité équivalente à celle des villes moyennes et des grandes villes! Ainsi, au quotidien - en matière d'offre de services de consommation - les petites villes pourvoient aux mêmes besoins que les grandes agglomérations avec moins de contraintes liées au trafic routier, à la difficulté de parking, etc. On voit, par ailleurs, que les villes moyennes et les grandes villes font « jeu égal » en matière de services intermédiaires. En, revanche, les petites villes décrochent. Enfin, les grandes villes se distinguent clairement des autres types en matière d'offre de services supérieurs.

Notre hypothèse est de considérer que plus l'offre d'équipements de commerces et de services est diversifiée, plus les points ou nœuds de croisement et de convergence de nos mobilités du quotidien sont structurants et plus le territoire maximise sa propension à faire circuler les richesses. La densité et la diversité de la présence d'équipements quotidiens confirment l'importance des petites villes et des gros bourgs-centres dans les mobilités quotidiennes, ces équipements sont des indicateurs de nœuds de convergence dans nos parcours quotidiens complexes et éclatés. Les petites villes structurent économiquement et socialement nos trajectoires individuelles et nos territoires. Il semble difficile, voire risqué et contreproductif de vouloir renforcer, re-densifier les métropoles à des fins économiques, environnementales sans identifier et intégrer ce résultat. L'objectif ou l'enjeu ne semble pas devoir être défini en termes de compétition et de concurrence, mais se pense en termes de complémentarité réciproque, de synergie positive entre métropoles, petites villes et hinterland.

\section{Géographie des équipements de la centralité des quotidiens}

Les cartes qui suivent, distinguent à l'échelle communale, le degré de diversité des services offerts par les territoires à la population, à savoir les équipements de centralité des quotidiens (figure 1 planche I), des services mobiles de la proximité (figure 2 - planche II), les équipements intermédiaires (figure 3 - planche III), les équipements supérieurs (figure 4 - planche IV).

Ces cartes montrent la présence et la diversité des équipements de centralité des quotidiens et des services mobiles de proximité sur l'ensemble du territoire. Les équipements intermédiaires se concentrent dans les villes, petites à moyennes, et les grandes villes, alors que l'offre d'équipements supérieurs apparaît plus concentrée et diversifiée dans les grandes villes.

La présence de communes non équipées est plus grande dans les espaces les moins denses comme dans les campagnes normandes, ou encore dans celles du Nord-Est, dans les Pyrénées. Ainsi, nous constatons une progression de la diversité des équipements de centralité quotidienne dans les territoires aux densités plus élevées (annexe 3). Mais ce lien entre offre et demande apparente, c'est-àdire entre diversité des équipements et densité de population n'est pas systématique. Il est intéressant de révéler les communes qui au regard de leur densité de population apparaissent comme sous ou suréquipées, toujours en termes de diversité des équipements de centralité du quotidien (figure 5 planche V).

Ainsi, les communes dont la diversité des équipements de centralité des quotidiens est plus importante que ce que suggère leur densité de population sont pour la plupart situées au sud d'une ligne reliant la Normandie à la Haute-Savoie. Dans cet espace, les territoires les mieux équipés sont le plus souvent des lieux à forte attractivité touristique et résidentielle à l'instar de la côte atlantique, du littoral et de l'arrière-pays méditerranéen, des Alpes... Inversement, cette offre est moindre que l'attendu 
théorique dans certains territoires situés dans l'Est, mais aussi dans le Nord ou le Nord-Ouest (Normandie, Picardie, Nord-Pas-de-Calais, dans les départements situés de la Moselle au Jura, mais également dans les espaces ruraux de la Gironde et de la Charente, ou encore au pied des Pyrénées).

Il y aurait donc une corrélation entre la diversité de l'offre d'équipements de centralité des quotidiens et l'attractivité résidentielle et touristique des territoires. La relation peut d'ailleurs être à double sens. L'arrivée de nouvelles populations résidentes ou présentes constitue un apport de richesse extérieure considérable, entraînant une hausse de la demande de biens et services courants. La présence d'une offre de structures et de services d'usage " quotidiens » peut, à son tour, constituer un « avantage comparatif », un atout auprès des populations candidates pour une installation résidentielle. C'est le point développé dans les pages qui suivent.

\section{Centralité des quotidiens ET DÉVELOPPEMENT LOCAL DES TERRITOIRES}

Cette corrélation entre la centralité des quotidiens et le processus de développement territorial, doit être mesurée et validée. Il s'agit d'apporter des éléments pour tester notre hypothèse selon laquelle la centralité des quotidiens - mesurée par la diversité de l'offre d'équipements - est un bon indicateur des nœuds d'échanges et de convergence de nos mobilités quotidiennes, des points d'ancrage collectifs structurants. Dans une perspective économique, nous souhaitons valider le fait que la centralité des quotidiens est un bon indicateur à la fois de circulation locale des richesses, mais également de l'attractivité résidentielle des territoires.

\section{L'interdépendance entre économie pro- ductive, résidentielle et présentielle, moteur du développement local}

L'augmentation du temps libre, l'allongement de la durée de vie, les progrès technologiques, l'amélioration des niveaux de revenus des ménages, etc., ont permis l'essor des mobilités et ont eu raison d'une logique qui associait un individu à un territoire. C'est la multi-appartenance territoriale des individus qui explique, qu'aujourd'hui, la production et la consommation demandent à être déterminées par des lois géographiques et économiques différentes. Cette déconnexion spatiale et temporelle de l'offre et de la demande a fait émerger un fait nouveau et sans précédent pour l'économie des territoires : le désajustement entre la création de richesse et l'amélioration des conditions de vie des populations. Ce désajustement entre croissance et développement au niveau local résulte de mécanismes de redistribution publique et privée entre les territoires. Ces flux de revenus constituent une ressource majeure et un levier d'action pour les territoires et leurs acteurs. Ces revenus, que l'on peut qualifier de base résidentielle en référence aux travaux sur le sujet (Davezies, 2008; Talandier et Davezies, 2009), influent fortement sur le développement économique, social et démographique local.

Ainsi, le développement économique d'un territoire dépend de sa capacité à capter ces flux de revenus, tout autant qu'à créer de la richesse. Mais le processus de développement ne s'arrête pas là. En effet, ces revenus captés et créés doivent ensuite être dépensés localement pour générer des emplois, induire de nouveaux revenus, attirer de nouvelles populations. L'enjeu du développement local repose donc sur un triptyque en interrelation, à savoir : créer des richesses (économie ou base productive); capter des richesses (économie ou base résidentielle et touristique); faire circuler ces richesses (secteur domestique ou sphère présentielle ${ }^{14}$ ) (figure 6). Il n'y a donc pas lieu d'opposer " économie productive, résidentielle ou présentielle ». Il est fondamental au contraire, de considérer que le fonctionnement des territoires repose sur ce triptyque, que l'équilibre entre ces trois mécanismes est facteur de dynamisme et, probablement, de développement durable. Ces trois temps du développement peuvent être concomitants, ou former les phases successives d'un cycle économique.

De la même façon qu'au jeu de captation des revenus, tous les territoires ne sont pas égaux, la propension à consommer localement varie d'un territoire à l'autre. L'existence d'un tissu préexistant de commerces, de services, d'infrastructures publiques et privées constituerait donc un atout à partir duquel peuvent se développer de nouvelles activités pour

14. En référence à la terminologie de l'INSEE qui utilise «sphère présentielle » afin de qualifier les emplois localisés destinés à répondre à la demande locale. 
Figure 6: Penser l'interdépendance entre économie productive, économie résidentielle et économie présentielle (Source : M. Talandier)

The interdependence between productive economy, residential economy and domestic economy

desservir la demande locale, mais également attirer une demande extérieure ou de nouveaux revenus basiques (Markusen, 2007 et 2009). Ainsi, l'effet économique de l'offre de centralité des quotidiens est double :

- elle maximise la propension à consommer localement et faire circuler les richesses dans les territoires;

- elle attire de nouvelles populations et de nouveaux revenus.

Le schéma du développement local (figure 7) permet de localiser ces deux effets dans le processus de développement local. Schématiquement, tout ce passe selon un mécanisme néo-classique en amont, et keynésien en aval. On trouve en amont du cycle de développement, les revenus basiques ou revenus captés à l'extérieur qui alimentent le modèle, plus particulièrement l'économie productive et résidentielle. Ce sont les moteurs de l'économie locale. En aval, la consommation est symbolisée par une «boîte noire ». En effet, rien en France à ce jour, ne permet de mesurer la consommation des ménages à l'échelle locale; l'enquête budget des ménages n'est pas territorialisée, des enquêtes locales et ponctuelles existent mais ne permettent pas un travail exhaustif.

Si l'on ignore ce qui se passe exactement en termes de consommation, on connaît plusieurs éléments susceptibles d'influencer la consommation en amont et d'en identifier l'impact en aval. En amont, la présence (touristique et journalière) détermine le niveau de la demande. La présence mesure la population effectivement présente dans le territoire à savoir la population recensée plus les soldes entrée-sortie de touristes et de navetteurs.

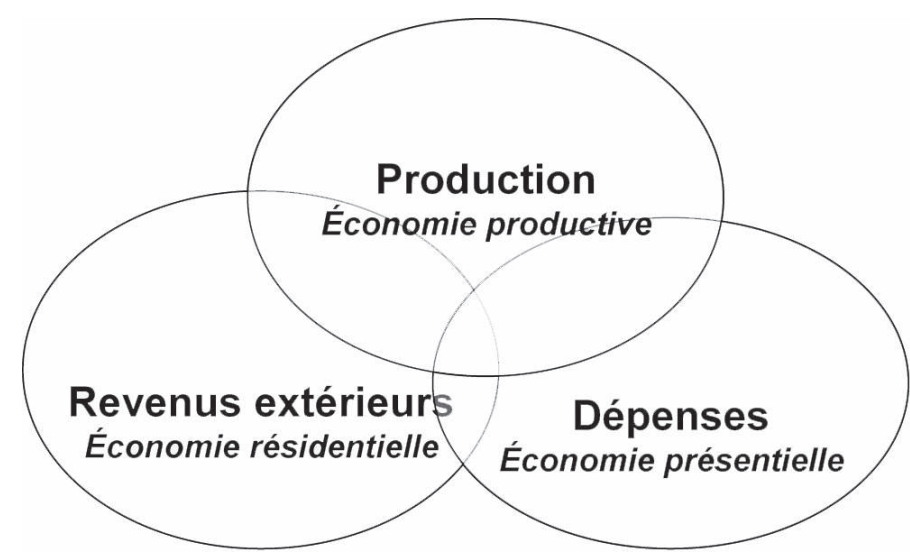

- Présence touristique $=$ population recensée + touristes - résidents absents (touristes ailleurs)

- Présence en journée $=$ population recensée + actifs travaillant dans le territoire mais n'y résidant pas - actifs résidents mais travaillant en dehors du territoire.

Plus la présence sur un territoire est importante, plus ce territoire a de probabilité de voir ces personnes consommer localement sous réserve que l'offre de commerces et services - notamment de centralité des quotidiens - soit suffisamment développée et offre une palette de choix satisfaisante.

Si le territoire capte des richesses et parvient à faire circuler ces richesses en «interne », cela se traduit par un essor (ou a minima, un maintien) des emplois dans les commerces, services, activités culturelles, associatives, récréatives, etc. Un réservoir d'emplois s'offre à la population locale, notamment peu qualifiée. Mais ces emplois peuvent, d'une part ne pas être occupés par les résidents, d'autre part entretenir une certaine fragilité sociale (emplois peu rémunérés, temps partiels, emplois saisonniers...).

L'essor des emplois domestiques ou présentiels induirait une diminution du chômage, une amélioration du niveau de vie et favoriserait la propension à consommer, créant ainsi une spirale cumulative de la demande (principe de développement local keynésien). L'essor de l'offre de commerces et de services influe sur l'attractivité du territoire - attractivité résidentielle, mais peut-être aussi productive, entretenant une spirale de développement de l'offre.

L'impact de l'offre de centralité des quotidiens sur le développement territorial serait donc double selon notre hypothèse. Cette offre pourrait être à la fois 


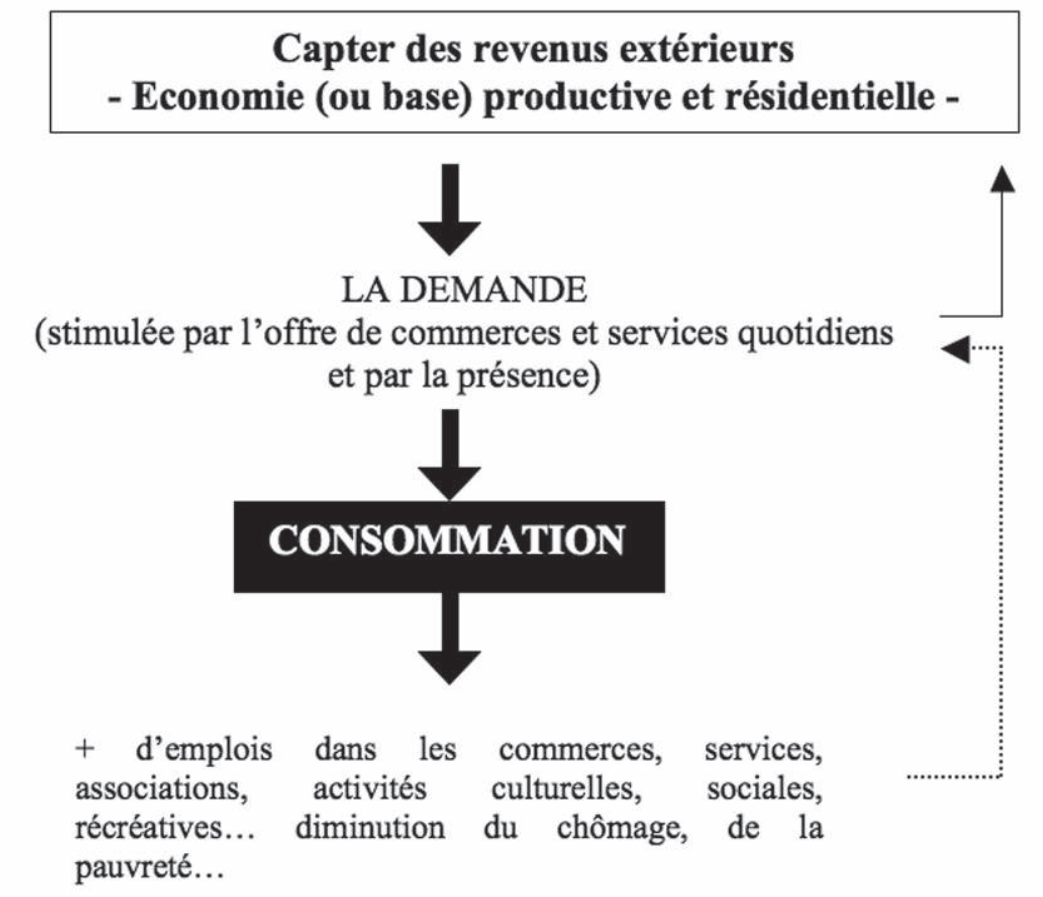

\author{
Enjeux de compétitivité et/ou de \\ différenciation productive mais \\ également enjeu d'attractivité \\ résidentielle et touristique \\ Enjeux de maximisation de la propension \\ à consommer localement
}

... spirale cumulative par la demande locale qui repose donc sur :

- la captation de revenus extérieurs (agir sur l'attractivité et la compétitivité)

- la propension locale à consommer (favoriser la présence et développer l'offre de commerces, services...)

- l'occupation des emplois domestiques par des résidents (organisation et gestion des emplois).

Figure 7 : Schéma du développement local (Source : M. Talandier) Local development scheme

un facteur d'attractivité de nouvelles populations et de nouveaux revenus et un facteur de circulation des richesses dans les territoires. C'est, en tout cas, ce que suggèrent les premières analyses que nous avons menés, dans le cas des petites villes, des bourgs-centres et des espaces ruraux.

\section{Centralité des quotidiens et circulation des richesses}

L'hypothèse selon laquelle l'offre d'équipements de centralité des quotidiens serait facteur de circulation de richesse, et participerait donc au développement économique local est testée ici à partir de premières analyses de corrélation. En effet, nous avons admis que plus la présence sur un territoire est importante, plus ce territoire a de probabilité de maximiser sa propension à consommer localement sous réserve qu'une offre diversifiée de commerces et services de centralité des quotidiens, existe. Nous avons également admis que si le territoire capte des richesses et parvient à faire circuler ces richesses en "interne », cela a un impact sur les emplois de la sphère présentielle (activités tournées vers la demande locale).Pour ces premières analyses, nous avons donc calculé les taux de présence touristique, les taux de présence en journée ainsi que les taux d'emplois «présentiels » pour 1000 habitants dans les communes françaises, avec:

- taux de présence touristique $=$ (présence touristique/population recensée) x 100 
Tableau 4 : Coefficient de corrélation entre l'offre d'équipements, les taux de présence et les emplois présentiels selon la hiérarchie urbaine (Source : INSEE, recensement 2007; BPE 2009; Insee 2008; ministère du Tourisme; Calculs : M. Talandier et V. Jousseaume)

La diversité des équipements est le nombre d'équipements quotidiens différents. La densité d'équipements quotidiens est le nombre total d'équipements pour 1000 hab. La taille des communes : villages et bourgs $<3000$ hab.; Bourg-centre ou petite ville : de 3000 à 20000 hab.; ville moyenne : de 20000 hab. à 100000 hab.; grande ville : plus de 100000 hab.

Correlation coefficient between the supply of services, rates of presence and domestic employment

\begin{tabular}{|c|c|c|c|}
\hline & $\begin{array}{c}\text { Coef. de } \\
\text { corrélation } \\
\text { entre taux } \\
\text { de présence } \\
\text { touristique et } \\
\ldots\end{array}$ & $\begin{array}{c}\text { Coef. de } \\
\text { corrélation } \\
\text { entre taux de } \\
\text { présence en } \\
\text { journée et ... }\end{array}$ & \begin{tabular}{|c} 
Coef. de \\
corrélation \\
entre la \\
densité des \\
emplois \\
présentiels et
\end{tabular} \\
\hline \multicolumn{4}{|l|}{... la diversité des équipements "quotidiens" } \\
\hline Gdes villes & 0,07 & 0,11 & 0,07 \\
\hline Villes moyennes & $-0,07$ & 0,16 & 0,11 \\
\hline Petites villes (ou bourgs centre) & 0,07 & 0,24 & 0,18 \\
\hline Villages et bourgs & 0,10 & 0,19 & 0,22 \\
\hline \multicolumn{4}{|l|}{... la densité d'équipements "quotidiens" } \\
\hline Gdes villes & 0,45 & 0,40 & 0,39 \\
\hline Villes moyennes & 0,59 & 0,46 & 0,41 \\
\hline Petites villes (ou bourgs centre) & 0,56 & 0,39 & 0,33 \\
\hline Villages et bourgs & 0,37 & 0,25 & 0,30 \\
\hline
\end{tabular}

- taux de présence en journée $=$ (présence en journée/population recensée) x 100

Nous avons ensuite calculé les coefficients de corrélation entre ces indicateurs, révélateurs de circulation de richesse, et les indicateurs d'offre d'équipements de centralité des quotidiens (intensité et diversité). Ces coefficients sont donnés dans le tableau 4, selon la hiérarchie urbaine.

En termes de densité d'équipements pour 1000 habitants, les coefficients de corrélation entre les différentes variables sont globalement et significativement positifs. L'offre d'équipements de centralités des quotidiens est corrélée à la présence effective des populations dans le territoire et favorise les activités, ou l'emploi présentiel. Ces quatre indicateurs, positivement et significativement corrélés, apparaissent comme des révélateurs de consommation et de circulation des richesses dans les territoires. Sur le plan de la diversité de l'offre quotidienne, les résultats sont plus contrastés que ce soit par type de villes ou par type d'indicateurs (présence touristique, présence à la journée et emplois présentiels). Ainsi, la présence touristique apparaît moins sensible à la diversité de l'offre (et inversement). Cela signifie que la consommation touristique se concentre sur une gamme peut-être plus réduite de services. Si tourisme et maintien ou essor des équipements « quotidiens » des territoires, semblent aller de pair, cette relation ne porte que sur un nombre restreint de services. Il n'y aurait pas, ou très peu, de lien entre la diversité de l'offre et l'intensité touristique du territoire.
En revanche, la présence en journée, d'actifs ou non, semble favoriser la diversité des équipements dans les petites villes et les bourgs-centres, mais aussi dans les villages ou communes de moins de 3000 habitants. La corrélation est beaucoup moins nette pour les niveaux supérieurs, villes moyennes ou grandes. Le même résultat est observé pour les emplois présentiels. Ces emplois sont plus représentés dans les petites villes et les campagnes qui offrent un éventail de commerces et services quotidiens plus large. Cette corrélation entre diversité et emplois « présentiels » est inexistante dans les villes moyennes ou les grandes villes.

Ces premiers résultats suggèrent que l'offre de centralité des quotidiens aurait un fort impact sur le développement territorial des petites villes et des campagnes, à travers les mécanismes de consommation ou de circulation des richesses. Mais, nous pensons que cette diversité de l'offre peut aussi être facteur d'attractivité et entretenir ainsi le processus de développement.

\section{Centralité des quotidiens et attractivité résidentielle de longue distance}

Afin de tester l'impact de l'offre de services et commerces quotidiens sur l'attractivité des territoires, nous avons prolongé un travail d'analyse des mobilités d'agrément (Talandier, 2012). Ce travail nous a permis de calculer le nombre de migrants nouvellement installés qui habitaient auparavant dans un pôle urbain, au sens INSEE. Nous avons 
distingué, au sein de ces nouveaux arrivants, ceux qui viennent d'un pôle urbain situé à moins de $100 \mathrm{~km}$ et ceux provenant d'un pôle urbain situé à plus de 100 km. On sait, en effet, que les comportements, stratégies, motivations, localisations de migrations sont tout à fait différents s'il s'agit d'une mobilité résidentielle du type «étalement urbain » ou s'il s'agit d'une mobilité dites d'agrément ou amenity migration (Hall et Williams, 2002 ; Nelson, 2004 ; Moss, 2006; Cognard, 2010). Dans le premier cas, le facteur économique est prédominant et les choix sont dictés par les différentiels : coût foncier/coût de l'éloignement au centre. Dans le cas des mobilités résidentielles d'agrément, les facteurs sont en premier lieu liés aux aménités paysagères et environnementales. L'envie de changer radicalement de cadre de vie est prégnante. Ainsi, nous distinguons les immigrants de « courte distance », des immigrants de «longue distance » qui ont décidé de quitter un pôle urbain pour s'installer dans une commune de l'espace à dominante rurale ${ }^{15}$.
La figure 8 nous indique la part des néo-ruraux (ceux qui vivaient dans un pôle urbain INSEE au recensement précédent) au sein de la population des communes de l'espace rural, en fonction de la diversité de leur offre d'équipements du quotidien. L'attractivité de ces communes auprès des migrants ayant quitté un pôle urbain semble proportionnelle à la diversité des équipements de centralité des quotidiens offerts dans la commune. Ainsi, ces migrants « urbains » installés depuis moins de cinq ans représentent près de $10 \%$ de la population des communes de moins de 20000 habitants, ayant une offre complète (22 équipements quotidiens présents). Ils ne représentent que $7 \%$ de la population, lorsqu'il n'y a qu'un service présent dans la commune. Certes ce différentiel de trois points de pourcentage peut sembler modeste, mais il constitue un premier résultat nous encourageant à approfondir notre recherche. En effet, comme cela a été démontré par d'autres auteurs (Baccaïni, 2007; Gonnard, 2012), les modèles migratoires se sont
Part en \% des néo-ruraux dans la population en 2007

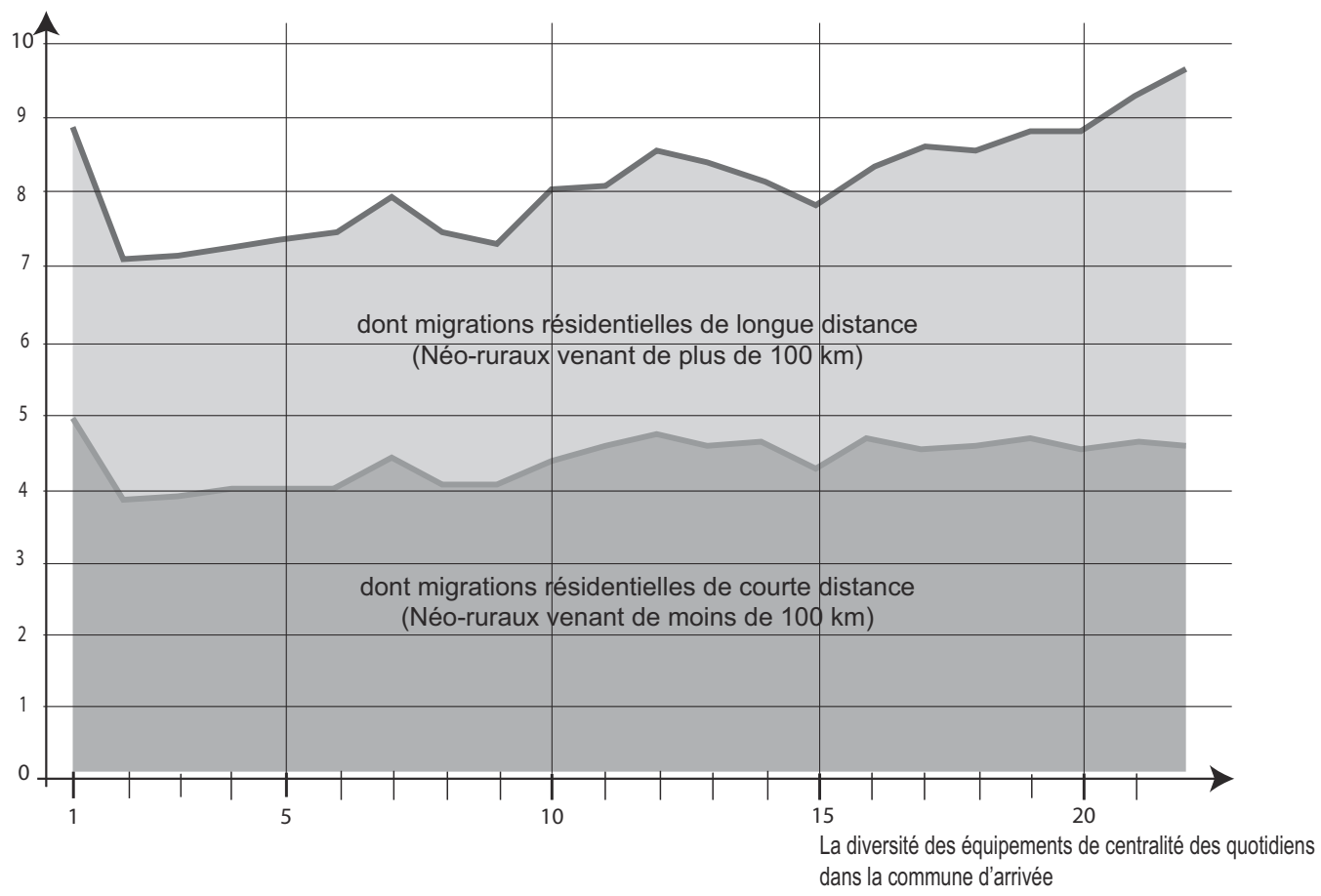

Figure 8 : Part des néo-ruraux dans la population des communes selon la diversité de leur offre d'équipements et services de centralité des quotidiens (Source : INSEE, BPE 2009 et recensement 2007 ; Réalisation : M. Talandier et V. Jousseaume) Immigrants share of urban centers in the population of rural areas according to the diversity of daily services

15. À savoir un espace constitué de petits bourgs ruraux (entre 1500 et 5000 emplois) et de communes peu ou pas sous influence urbaine (ni pôle urbain, ni périurbain). Il s'agit du zonage en aire urbaine antérieur à celui de 2010 , non disponible au moment du calcul de ces migrations résidentielles de courte et longue distance sur données 2007. 
inversés à partir des années 1970. Les régions les plus attractives en France n'étant plus nécessairement les régions les moins riches économiquement. La sensibilité à cette offre d'équipements varie selon que les migrations se font sur une courte ou une longue distance. On constate une corrélation plus marquée entre l'offre de services de centralité des quotidiens et la part des immigrants de longue distance.

L'indicateur de centralité des quotidiens proposé dans cet article apparaît donc positivement corrélé à l'attractivité résidentielle des espaces non urbains (bourgs ruraux et communes plus isolées). Par ailleurs, les migrants de longue distance semblent plus sensibles à la présence de ces services que les migrants périurbains, poursuivant des stratégies résidentielles distinctes.

\section{Conclusion}

En dehors des mobilités de travail qui ne concernent que la moitié de la population et moins du quart des déplacements des actifs eux-mêmes, les mobilités banales, quotidiennes, de la population française ne sont pas connues, car complexes et non mesurées par les institutions publiques produisant de la statistique. Or, l'observation des points de convergence élémentaires de nos mobilités banales, à travers l'offre d'équipements de proximité, laisse apparaître le maillage dense et serré des centralités de nos quotidiens depuis le village-centre élémentaire jusqu'aux grandes villes.

Les premiers résultats de cette réflexion qui s'engage, avancent plusieurs apports importants que nous résumons. Premier constat, en termes de densité d'équipements quotidiens, il n'y a pas de différences significatives entre l'urbain et le rural, entre les grandes villes et les bourgs. En termes de diversité de l'offre du quotidien, le rural cette fois décroche, l'indicateur met en exergue le rôle éminemment structurant des villes. Toutefois, il est remarquable d'observer que les petites villes et les gros bourgs-centres (3000 à 20000 habitants) offrent une diversité de choix en matière de centralité des quotidiens, équivalente à celle des villes moyennes et des grandes villes.

Deuxième résultat, l'offre de centralité des quotidiens est corrélée à la population présente dans le territoire (touristes ou actifs) qui détermine les mécanismes de consommation et de circulation de richesses. Cette relation est moins nette pour les villes moyennes et grandes.

Troisième résultat, la diversité de l'offre satisfaisant les besoins les plus banals, est un facteur d'attractivité résidentielle et donc de captation de revenus. L'offre de centralité des quotidiens semble constituer un facteur important du développement des territoires en général et des petites villes, des bourgs-centres et des campagnes en particulier.

Ce bilan nous parait encourageant et stimulant pour approfondir la réflexion, par exemple en détaillant les mobilités résidentielles (profil des migrants, géographie...) ou encore en étudiant l’impact de «bouquets » de services divers pour repérer les services particulièrement structurants et attractifs selon les populations. Ces premiers travaux ouvrent des perspectives de recherche sur un thème émergeant et très peu investi : celui de la relation entre la consommation et les territoires. 


\section{Annexe 1 : Gammes d'équipements pro- posés par l'IINSEE, Base Permanente des Équipements 2009}

\begin{tabular}{|c|c|c|}
\hline Gamme Proximité & Gamme Intermédiaire & Gamme Supérieure \\
\hline Banque, caisse d'épargne & Police, gendarmerie & Pôle emploi \\
\hline Bureau de poste, relais poste, agence postale & Trésorerie & Location d'automobiles et d'utilitaires légers \\
\hline Réparation automobile et de matériel agricole & Pompes funèbres & Agence de travail temporaire \\
\hline Maçon & Contrôle technique automobile & Hypermarché \\
\hline Plâtrier, peintre & Ecole de conduite & Produits surgelés \\
\hline Menuisier, charpentier, serrurier & Vétérinaire & Poissonnerie \\
\hline Electricien & Supermarché & Lycée d'enseignement général et/ou technologique \\
\hline Entreprise générale du bâtiment & Librairie, papeterie & Lycée d'enseignement professionnel \\
\hline Coiffeur & Magasin de vêtements & Etablissement de santé de court séjour \\
\hline Restaurant & Magasin d'équipements du foyer & Etablissement de santé de moyen séjour \\
\hline Agence immobilière & Magasin de chaussures & Etablissement de santé de long séjour \\
\hline Soins de beauté & Magasin d'électroménager & Etablissement psychiatrique \\
\hline Epicerie, supérette & Magasin de meubles & Urgences \\
\hline Boucherie, charcuterie & Droguerie, quincaillerie, bricolage & Centre de santé \\
\hline Fleuriste & Horlogerie, bijouterie & Structures psychiatriques en ambulatoire \\
\hline Ecole maternelle & Collège & Spécialiste en cardiologie \\
\hline Ecole élémentaire & Opticien, lunetier & Spécialiste en dermatologie et vénéréologie \\
\hline Médecin omnipraticien & Orthophoniste & Spécialiste en gynécologie médicale \\
\hline Chirurgien dentiste & Pédicure, podologue & Spécialiste en gynécologie obstétrique \\
\hline Infirmier & Laboratoire d'analyses médicales & Spécialiste en gastro-entérologie, hépatologie \\
\hline Masseur kinésithérapeute & Ambulance & Spécialiste en psychiatrie \\
\hline Pharmacie & Personnes âgées : hébergement & Spécialiste en ophtalmologie \\
\hline Taxi & Personnes âgées : soins à domicile & Spécialiste en oto-rhino-laryngologie \\
\hline Boulodrome & Personnes âgées : service d'aide & Spécialiste en pédiatrie \\
\hline Tennis & Garde enfants d'âge préscolaire & Spécialiste en radiodiagnostic et imagerie médicale \\
\hline Salle ou terrain multisports & Bassin de natation & Sage-femmes \\
\hline & Roller, skate, vélo bicross ou freestyle & Enfants handicapés : soins à domicile \\
\hline & & Adultes handicapés : hébergement \\
\hline & & Adultes handicapés: services \\
\hline & & Travail protégé \\
\hline & & Cinéma \\
\hline
\end{tabular}




\begin{tabular}{|c|c|c|c|}
\hline Nom & $\begin{array}{c}\text { Nb de } \\
\text { communes } \\
\text { équipées }\end{array}$ & $\begin{array}{c}\text { Part des } \\
\text { communes } \\
\text { équipées } \\
(\%)\end{array}$ & Gamme Insee \\
\hline Ecole élémentaire & 22749 & 62,1 & proximité \\
\hline Maçon & 19163 & 52,3 & proximité \\
\hline Menuisier, charpentier, serrurier & 18559 & 50,7 & proximité \\
\hline Terrains de grands jeux & 18096 & 49,4 & proximité \\
\hline Plombier, couvreur, chauffagiste & 18048 & 49,3 & proximité \\
\hline Restaurant & 17452 & 47,7 & proximité \\
\hline Plâtrier peintre & 15867 & 43,3 & proximité \\
\hline Electricien & 15301 & 41,8 & proximité \\
\hline Réparation automobile et de matériel agricole & 15038 & 41,1 & proximité \\
\hline Boulodrome & 14027 & 38,3 & proximité \\
\hline Coiffure & 13605 & 37,2 & proximité \\
\hline Tennis & 13580 & 37,1 & proximité \\
\hline Boulangerie & 13480 & 36,8 & proximité \\
\hline Salle non spécialisé & 11079 & 30,3 & R. \\
\hline Plateau extérieur ou salle multisports & 10356 & 28,3 & R. \\
\hline Infirmier & 10291 & 28,1 & proximité \\
\hline Médecin omnipraticien & 10118 & 27,6 & proximité \\
\hline Bureau de poste & 9478 & 25,9 & proximité \\
\hline Agence immobilière & 8889 & 24,3 & proximité \\
\hline Pharmacie & 8368 & 22,9 & proximité \\
\hline Epicerie & 8360 & 22,8 & proximité \\
\hline Taxi & 8356 & 22,8 & proximité \\
\hline Boucherie charcuterie & 8205 & 22,4 & proximité \\
\hline Masseur kinésithérapeute & 8047 & 22,0 & proximité \\
\hline Ecole maternelle & 7844 & 21,4 & proximité \\
\hline Entreprise générale du bâtiment & 7254 & 19,8 & proximité \\
\hline Chirurgien dentiste & 6498 & 17,7 & proximité \\
\hline Banque, Caisse d'Epargne & 6373 & 17,4 & proximité \\
\hline Fleuriste & 6286 & 17,2 & proximité \\
\hline Salle de terrains de petits jeux & 6223 & 17,0 & proximité \\
\hline Soins de beauté & 6144 & 16,8 & proximité \\
\hline Hôtel homologué & 5732 & 15,7 & N.C \\
\hline Personnes âgées : hébergement & 5496 & 15,0 & intermédiaire \\
\hline Camping homologué & 5036 & 13,8 & N.C \\
\hline Librairie papeterie journeaux & 4945 & 13,5 & intermédiaire \\
\hline Ecole de conduite & 4562 & 12,5 & intermédiaire \\
\hline Magasin de vêtements & 4540 & 12,4 & intermédiaire \\
\hline Supermarché & 4490 & 12,3 & intermédiaire \\
\hline Personnes âgées : services d'aide & 4178 & 11,4 & intermédiaire \\
\hline Orthophoniste & 4025 & 11,0 & intermédiaire \\
\hline Vétérinaire & 4012 & 11,0 & intermédiaire \\
\hline Centre équestre & 4009 & 11,0 & N.C \\
\hline Salle de combat & 4000 & 10,9 & R. \\
\hline Ambulance & 3972 & 10,8 & intermédiaire \\
\hline Pédicure-podologue & 3881 & 10,6 & intermédiaire \\
\hline Pompes funèbres & 3771 & 10,3 & intermédiaire \\
\hline Droguerie quincaillerie bricolage & 3759 & 10,3 & intermédiaire \\
\hline Collège & 3703 & 10,1 & intermédiaire \\
\hline Magasin d'articles de sports et de loisirs & 3584 & 9,8 & intermédiaire \\
\hline etc ... & $\ldots$ & $\ldots$ & $\ldots$ \\
\hline Horlogerie Bijouterie & 2068 & 5,6 & intermédiaire \\
\hline Laboratoire d'analyses médicales & 1983 & 5,4 & intermédiaire \\
\hline Parfumerie & 1855 & 5,1 & supérieure \\
\hline Personnes âgées : soins à domicile & 1831 & 5,0 & intermédiaire \\
\hline Parcours sportif & 1791 & 4,9 & N.C \\
\hline Lycée d'enseignement professionnel & 1681 & 4,6 & supérieure \\
\hline Sports nautiques & 1662 & 4,5 & N.C \\
\hline Agence de travail temporaire & 1423 & 3,9 & supérieure \\
\hline Etablissement santé moyen séjour & 1396 & 3,8 & supérieure \\
\hline Poissonnerie & 1367 & 3,7 & supérieure \\
\hline Centre de santé & 1351 & 3,7 & supérieure \\
\hline Enfants handicapés : hébergement & 1329 & 3,6 & supérieure \\
\hline Travail protégé & 1318 & 3,6 & supérieure \\
\hline Sage-femme & 1286 & 3,5 & supérieure \\
\hline Location automobiles, utilitaires légers & 1279 & 3,5 & supérieure \\
\hline Spécialiste en radiodiagnostic et imagerie médical & 1244 & 3,4 & supérieure \\
\hline Spécialiste en ophtalmologie & 1232 & 3,4 & supérieure \\
\hline Cinéma & 1178 & 3,2 & supérieure \\
\hline etc... & $\ldots$ & $\ldots$ & $\ldots$ \\
\hline
\end{tabular}

\section{Annexe 2 : Équipements les plus courants en France métropoli- taine : nombre et part de com- munes équipées en 2009}

(N.C. : équipement "non considéré » dans les gammes INSEE; R. : équipement regroupé avec un autre équipement; Source : calculs M. Talandier, d'après INSEE, BPE 2009) 


\begin{tabular}{|l|c|}
\hline $\begin{array}{c}\text { Densité de population } \\
\text { en 2009 (hab./km²) }\end{array}$ & $\begin{array}{c}\text { Diversité des } \\
\text { équipements de centralité } \\
\text { des quotidiens }\end{array}$ \\
\hline$[0-10,1[$ & 1,5 \\
\hline$[0-15,5[$ & 2,3 \\
\hline$[0-21,3[$ & 3,0 \\
\hline$[0-28,9[$ & 3,6 \\
\hline$[0-38,8[$ & 4,6 \\
\hline$[0-52,4[$ & 5,7 \\
\hline$[0-73,9[$ & 6,9 \\
\hline$[0-113,1[$ & 8,9 \\
\hline$[0-227,6[$ & 11,8 \\
\hline$>=227,6$ & 17,4 \\
\hline
\end{tabular}

\section{Bibliographie}

Allen P., Sanglier M., 1979. A dynamic model of growth in a central place system, Geographical Analysis, p. 256-272.

Ascher F., 2009. L'âge des métapoles, Éditions de l’Aube, 389 p.

Baccaini B., 2007, Les flux migratoires interrégionaux en France depuis cinquante ans, Population, Vol. 62, p. 143-160.

Blanc M., Schmitt B. et Ambiaud E., 2007. Orientation économique et croissance locale de l'emploi dans les bassins de vie des bourgs et petites villes, Économie et Statistique, $n^{\circ} 402,18$ p.

Cognard F., 2010. Migrations d'agrément et nouveaux habitants dans les moyennes montagnes françaises: de la recomposition sociale au développement territorial. L'exemple du Diois, $d u$ Morvan et $d u$ Séronais, Thèse de doctorat en géographie, MSH - Clermont-Ferrand, 528 p.

Davezies L., 2008. La République et ses territoires. La circulation invisible des richesses, Paris, Seuil, coll. « La République des idées », $113 \mathrm{p}$.

Davezies L., Savy M., Talandier M., 2008. Développement productivo-résidentiel et mobilités dans les territoires du Limousin, Rapport pour la DRE du Limousin, 37 p.

Dugot P., Pouzenc M. (dir.), 2010. Territoires du commerce et développement durable, Paris, L'Harmattan, coll. « Itinéraires géographiques », $241 \mathrm{p}$.

Friedland J., Savy M., Talandier M., 2009. État des lieux et prospectives de l'offre et de la demande de piscines, étude pour le ministère de la Jeunesse, des Sports, de la Santé et de la Vie Associative, 189 p.

Gasnier A. (dir.), 2010. Commerce et ville ou commerce sans la ville? Entre logiques entrepreneuriales et politiques territoriales de développement durable, Presses Universitaires de Rennes, coll. « Espaces et territoires », 310 p.

Gonnard S., 2006. L'inversion des flux migratoires interrégionaux : de nouveaux rapports entre migrations internes et développement territorial?, Thèse de doctorat, CRETEILInstitut d'Urbanisme de Paris, Université Paris-XII, 375 p.

\section{Annexe 3 : Densité de population et diversité des équipements de centralité des quotidiens dans les communes fran- çaises en 2009}

(Source : calculs M. Talandier, d'après INSEE, Recensement et BPE 2009)

Gwiazdzinski L., 2007. Redistribution des cartes dans la ville malléable, Espace, Populations, Sociétés, n²-3, p. 397-410.

Hall C. M. et Williams A. (eds), 2002. Tourism and Migration: New Relationships between - Production and Consumptio, Dordrecht, Netherlands, Kluwer, 189 p.

Hilal M., Piguet V., Schmitt B., 1995. Communes rurales et petites villes dans la hiérarchie urbaine, Économie et statistique, $\mathrm{n}^{\circ} 282$, p. 21-36.

INRA, 2008. Les nouvelles ruralités à l'horizon 2030 : des relations villes-campagnes en émergence?, Paris, Quae, 112 p.

Jousseaume V., 1996. Les bourgs-centres à l'ombre d'une métropole l'exemple de la Loire-Atlantique, Thèse de doctorat, Université de Nantes, 591 p.

KAYSER, B., 1972. Un système d'information sur les petites villes françaises, Travaux et documents du CIEU, $125 \mathrm{p}$.

Laborie, J.-P., 1979. Les petites villes. Paris, CNRS, 311 p.

Le Jeannic T., Roussel P. François D. (coord.), 2010. La mobilité des Français. Panorama issu de l'enquête nationale transports et déplacements 2008, coll. « La Revue » du Service de l'Observation et des Statistiques (SOeS) du Commissariat Général au Développement Durable (CGDD), $228 \mathrm{p}$.

Markusen A., 2007. A Consumption Base Theory of Development: An Application to the Rural Cultural Economy, Agricultural and Resource Economics Review, p. 1-13.

Markusen A., 2009. Consumption-Driven Urban Development, Urban Geography, vol. 30, n 4, p. 344-367.

Moss L. (dir.), 2006. The Amenity Migrants: seeking and sustaining mountains and their cultures, ed. Lawrence Moss, Wallingford, UK CABI Press., 231 p.

Nelson P. B., 2004. Nonearnings income migration in the United States: anticipating the geographic impact of baby boom retirement, Center for Retirement Research, Boston College, Summary report for Sandell Retirement Research Grant, 66 p.

Orfeuil J.-P., 2008. Une approche lä̈que de la mobilité, Paris, Éditions Descartes \& Cie, 173 p. 
Pumain D., 1992. Les systèmes de villes, dans Bailly A., Ferras R., Pumain D., Encyclopédie de géographie, Paris, Economica. chap. 34, p. 645-664.

Pumain D., 1994. Hiérarchie urbaine, dans Encyclopédie d'économie spatiale, Économica, p. 333-342.

Pumain D., 2004. L'invention permanente : la hiérarchie urbaine, dans Bridel P. (ed.) Mélanges en l'honneur de Giovanni Busino, Genève, Labor et Fides, p. 66-86.

Sasken S., 1991. The global city: New York, London, Tokyo, Princeton University Press, $447 \mathrm{p}$.

Soumagne J. (dir.), 2002. Commerce et espaces fragiles. Essais sur la revitalisation en milieu urbain et rural, CNRS, coll. "Commerce et société », 200 p.

Talandier M., 2005. Les activités de la Poste face aux nouvelles dynamiques des territoires: Performance postale/performance territoriale, Rapport pour la direction du géomarketing de La Poste, 179 p.
TAlandier M., 2009. Une autre géographie du développement rural. Une approche par les revenus, Géocarrefour, Vol. 83-4, p. 259-267.

Talandier M., Davezies L., 2009. Repenser le développement territorial? Confrontation des modèles d'analyse et des tendances observées dans les pays développés, La Documentation française, coll. «Puca/Recherche », 144 p.

TAlandier M., 2012, L'impact territorial des migrations d'agrément en France, dans Bourdeau P., Niels M. (dir.), PostTourisme et migrations d'agrément, Paris, L'Harmattan, $412 \mathrm{p}$.

Terrier C., Sylvander M., Khiati A., 2005. En haute saison touristique, la population présente double dans certains départements, INSEE Première, n 1050, 4 p.

Viard J., 2004. Le sacre du temps libre, Paris, Édition de l'Aube, $220 \mathrm{p}$. 